\title{
Experimental Treatment with a Hypomethylating Agent Alters Life History Traits and Fitness in Brassica rapa
}

\author{
Emily J. Kottler $\mathbb{D}^{1},{ }^{1}$ Acer VanWallendael, ${ }^{2}$ and Steven J. Franks $\mathbb{D}^{2}$ \\ ${ }^{1}$ Department of Biological Sciences, The George Washington University, Washington, DC 20052, USA \\ ${ }^{2}$ Department of Biological Sciences, Fordham University, Bronx 10458, USA \\ Correspondence should be addressed to Steven J. Franks; franks@fordham.edu
}

Received 14 June 2018; Revised 26 September 2018; Accepted 22 October 2018; Published 1 November 2018

Academic Editor: Jutta Ludwig-Müller

Copyright ( 2018 Emily J. Kottler et al. This is an open access article distributed under the Creative Commons Attribution License, which permits unrestricted use, distribution, and reproduction in any medium, provided the original work is properly cited.

\begin{abstract}
Epigenetic modifications to DNA can be inherited and may play a key role in evolution, with epigenetic influences on life history traits such as the timing of germination and flowering thought to be particularly important in plants. However, few studies have examined the effects of epigenetic modifications through experimental alteration of the methylome under differential growth conditions. In this study, we chemically induced global DNA hypomethylation using 5-azacytidine in three Brassica rapa plant populations differing in life history characteristics and under differing photoperiod treatments. We found that our 5 azacytidine treatment affected the timing of germination and that this effect differed across populations, with the treatment delaying germination in B. rapa Fast Plants, which have been artificially selected for rapid cycling, but accelerating germination in biennials. Rapid cycling B. rapa plants also were smaller and had lower reproductive fitness under the experimental demethylation treatment. There was no main effect of demethylation or photoperiod on flowering time, but the interaction was marginally significant, indicating that demethylation effects on flowering time depend on photoperiod. These results demonstrate that epigenetic modifications can influence phenotypic traits in ways that are dependent on genetic identity, life history, and light availability.
\end{abstract}

\section{Introduction}

Understanding the mechanisms of evolution in natural populations is a fundamental goal in evolutionary biology and is critical for understanding how species can adapt to global change [1]. There is increasing recognition that evolution can occur through mechanisms beyond base-pair changes [2]. Epigenetic modifications, such as methylation of cytosines and histones, can alter gene expression, impact phenotypes and fitness, and can also be inherited [3-7]. For plants in particular, methylation changes to DNA and histones can have wide-ranging effects [8]. However, the specific role that methylation plays in regulation of ecologically relevant phenotypes is still being explored [9-11].

The term epigenetics refers to the heritable set of molecular processes that impact an organism's phenotype without changes to the DNA sequence, including primarily DNA methylation and histone modification [8]. DNA methylation, whereby the $5^{\prime}$ carbon of the cytosine aromatic ring gains a methyl group through the action of DNA methyltransferase, appears to be an important factor in the regulation of gene expression $[8,12,13]$. Methylation is important in controlling transposable elements (TEs) in several species [14-16] and regulating expression of subgenomes in polyploid species [17]. In addition, widespread accessions of Arabidopsis have shown a wide range of methylation patterns, and geographic specificity, indicating that methylation may be important at the population scale [8]. Further, methylation has been linked to local adaptation in Arabidopsis across Europe [18]. However, distinguishing DNA sequence and epigenetic evolution remains an experimental challenge outside Arabidopsis, and it remains unknown how methylation changes will play a role in adaptation to global change.

One of the primary responses to global change seen in a wide variety of species is a shift in the timing of life history events such as flowering time and other phenological traits $[19,20]$. The degree to which these phenotypic responses represent adaptive evolution or phenotypic plasticity remains 
an active area of investigation, with evidence for both evolution and plasticity found in several studies (reviewed in [21]). Previous work has shown evidence for adaptive evolution in flowering time and other traits in Brassica rapa (field mustard) in response to drought [22]. A recent study of these same populations showed extensive changes in allele frequencies across the genome, with some of the evolutionary changes in genes linked to flowering time and drought response [23]. However, it remains unknown if epigenetic changes could also have contributed to this rapid evolutionary shift in flowering time, which is a trait known to be influenced by epigenetic modifications [24]. Brassica rapa has also evolved via artificial selection, which was used to create rapid cycling fast plant cultivars that flower in only 14 days [25]. This life history pattern contrasts with biennial B. rapa varieties, which take two years, including a period of vernalization, to initiate flowering. This species is prime for this research as a previous study confirmed with Methylation-Sensitive Amplified Polymorphism profiling that treatment with 5azacytidine results in altered DNA methylation in Brassica rapa [26]. They found that in some B. rapa populations, the 5 -azaC demethylation treatment altered plant size, and this change in size was heritable, but they did not examine life history changes or environmental interactions.

One approach to studying epigenetics is to map out existing methylation patterns in the genome and compare these patterns across populations and species [27]. This method is fruitful, but it is resource intensive as it requires high-resolution reference genome assemblies and bisulfite sequencing methods to generate many individual methylomes, and it is descriptive rather than experimental. Another approach to directly assess responses of organisms to epigenetic modifications is to experimentally alter the methylome.

An agent commonly used in this experimental approach is 5-azacytidine (5-azaC), which has been consistently shown to alter nucleotide methylation patterns across the genome [28-30]. 5-Azacytidine blocks the active site of DNA methyltransferase enzymes which confer methylation patterns to the novel strand during DNA replication, inhibiting the methylation transferral process [28]. Administration of 5-azaC at the pregermination stage has been shown to directly reduce 5 -methylcytosine content in the genomic DNA of mature plants [31] as well as induce heritable phenotypic change [32]. However, it is important to note that when used at high concentrations, 5-azaC is incorporated into DNA and RNA causing cell death and not necessarily DNA demethylation, so due caution must be used in the interpretation of results generated from this methodological approach [33]. Despite the rather crude nature of this method, it is nevertheless advantageous because it allows researchers to quickly and easily generate populations that are genetically identical but epigenetically variant and perform manipulative experiments with these lines.

Previous studies have found that 5-azaC induced demethylation altered development rates and flowering time, two particularly important life history traits related to climate adaptation, in Arabidopsis thaliana [12], Linum usitatissimum [34], and Thlaspi arvense [35]. Furthermore, it has been shown that 5-azaC treated Arabidopsis thaliana presents not only DNA demethylation, but altered expression of FLC and FWA, two genes responsible for plant flowering [36].

In this study, we experimentally altered the methylome of $B$. rapa varieties that differed in life history characteristics. We then grew these populations under differing photoperiod conditions, as it has previously been shown that DNA demethylation via 5-azaC can impact flowering under differing light conditions [31]. We investigated the effects of this methylation treatment and photoperiod on the timing of germination and flowering, as well as on traits such as size and growth rate. We predicted that if methylation patterns are important for determining responses of plants to environmental signals that influence the timing of germination and flowering, the demethylation treatment would alter these traits and would have differential effects on populations with different life history characteristics. We further predicted that demethylation might induce flowering under conditions in which flowering normally does not occur, including under short days and in biennials before vernalization.

\section{Methods}

2.1. Study System. The study species is Brassica rapa L. (Brassicaceae), commonly known as field mustard. The species originated in the Middle East, but has spread widely throughout Europe, North America and Asia and has become naturalized in many areas. In agriculture, B. rapa has been cultivated to create a number of vegetables such as turnips, bok choy, and Chinese cabbage. B. rapa is also used extensively in laboratory research. Fast Plant cultivars have been artificially selected to undergo a full lifecycle in 30-40 days [25].

We studied three populations of $B$. rapa. Two of the populations are annual plants that flower within one year and do not require a vernalization treatment to flower. These are a Fast Plant cultivar from the Wisconsin Fast Plants Program and a naturalized population collected from the Back Bay site in the Upper Newport Bay Nature Preserve in Newport Beach, California in 2008 (permit \#19699-21901 from the UC Reserve System RAMAS). The third is an agricultural population from the Netherlands that is biennial (flowering once every two years) which was obtained from the USDA National Genetic Resources Program, via the Germplasm Resources Information Program (GRIN). This cultivar normally requires a vernalization treatment to flower, but plants were not vernalized in this study to determine if hypomethylation could replace vernalization.

2.2. Experimental Design. We used a full factorial design, with hypomethylation (demethylated or control), photoperiod (24hr or $8 \mathrm{hr}$ ), and population (California, Fast plant, or biennial) as factors. For each population, we randomly assigned 80 plants to one of each combination of methylation treatment and photoperiod, with 20 replicates for each.

For hypomethylation, we used the global demethylating agent 5-azacytidine (5-azaC), which is an effective inhibitor of methyltransferase, the enzyme that methylates newly synthesized DNA $[29,37]$. We cold-stratified 100 seeds from each of our three B. rapa populations (annual Californian, annual Fast Plant, and biennial Dutch) at $1-4^{\circ} \mathrm{C}$ in petri dishes. 
We placed half of each population on filter paper soaked with $1.4 \mathrm{~mL}$-distilled water, and half on filter paper with $1.4 \mathrm{~mL}$ of a $500 \mu \mathrm{mol} 5$-azaC solution (a concentration on the high-end of the spectrum used in previous studies using 5-azaC for demethylation; [12]). After five days of cold-stratification, we moved the plants to room temperature to germinate.

Once the seeds germinated, we transplanted seedlings into $6.5 \mathrm{~cm}^{2}$ pots in trays positioned in temperature and light-controlled growth chambers so that seedlings were 5$10 \mathrm{~cm}$ below the fluorescent lights, as per Fast Plant protocol (www.fastplants.org). We watered plants daily and fertilized twice per week with balanced NPK (20-20-20) fertilizer. We kept the growth chambers at $22^{\circ} \mathrm{C}$.

We recorded the dates of first observed budding, flowering, and senescence. To assess plant size, we noted the number of leaves and measured the length and width of the longest leaf at budding and flowering. Plants that did not flower by the end of the experiment had leaf number and size traits recorded at 6 weeks after germination.

2.3. Statistical Analysis. We determined the effects of hypomethylation treatment and population for time to germination with an ANOVA using SYSTAT v. 13 (Systat, Chicago, IL, USA). We further assessed differences between the treated plants and controls within each population using planned orthogonal contrasts in $\mathrm{R}$ version 3.2.2 (R Core Team 2014). We did not assess photoperiod for germination because all seeds were germinated under identical conditions of 24 hours/day of light. We transformed data as needed to meet model assumptions. We assessed the effects of the hypomethylation treatment, photoperiod, and variety on plant size characters using ANOVA. As the Fast Plant variety was the only population to flower with a sufficient sample size, and the biennial size data was collected at a different time than the Fast Plants, we also performed separate ANOVAs for all measured traits within these groups. Results were considered significant for $\mathrm{p}<0.05$ and marginally significant for 0.05 $<\mathrm{p}<0.10$. We confirmed results for all traits related to timing (time to germination, flowering time) with survival time analysis using a Cox proportional hazards model.

\section{Results}

3.1. Germination. Both the Fast Plants and biennials germinated at relatively high rates ( $\sim 80 \%$ each), while germination rate was low for the California population (15\%). Fast Plants were the earliest to germinate, followed by the biennials and then the California population (Figure 1).

Populations differed significantly in germination time $(p<0.001)$, but there was no significant main effect of 5 azaC on germination time $(\mathrm{p}=0.968)$ (Table 1$)$. However, we found a significant interaction between population and 5 -azaC treatment $(\mathrm{p}=0.002)$. Planned orthogonal contrasts showed that demethylation significantly increased time to germination in Fast Plants $(\mathrm{p}<0.05)$, with demethylated plants germinating about a day later than control plants, and marginally significantly decreased time to germination in for Biennials $(\mathrm{p}=0.0557)$, with control plants flowering less than a day later than treated plants (Table 1; Figure 1). There was

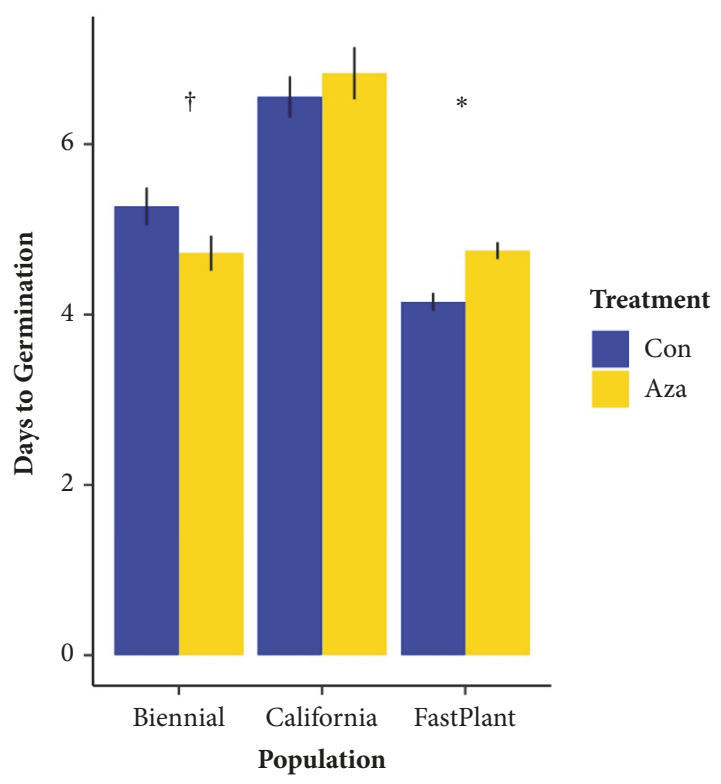

FIGURE 1: Mean number of days from methylation treatment to germination in three varieties of Brassica rapa. Blue bars (left) indicate control individuals ("Con") while yellow bars (right) denote individuals under 5-azacytidine hypomethylation treatment ("Aza”). Varieties have differing source locations and flowering regimes. Biennial: Netherlands, biennial; California: California, annual; FastPlant: Lab-selected, rapid annual. Error bars represent 1 SE. The following symbols denote significance, quantified by planned orthogonal contrast between "Aza" and "Con": ${ }^{\dagger} p<0.1, * p$ $<0.05$.

no significant effect of the 5 -azaC treatment on germination in the California population $(\mathrm{p}=0.487)$.

3.2. Growth and Flowering Time. The plant populations differed significantly in number of leaves and the width and length of the longest leaf (Table 2). All three of these size traits were also significantly affected by photoperiod (Table 2 ). There was not a significant effect of 5-azaC demethylation on number of leaves or longest leaf length, but there was a marginally significant $(\mathrm{p}<0.1)$ effect of 5 -azaC on longest leaf width (Table 2). There was a significant interaction between population and photoperiod for number of leaves and leaf length (Table 2).

In the Fast Plants (the only variety with a large proportion of individuals flowering during the study), photoperiod had a significant effect on number of leaves, length and width of the longest leaf, and flowering time, but not on number of flowers (Table 3).

Methylation treatment alone did not have a statistically significant effect on flowering time; however the interaction between methylation treatment and photoperiod treatment on flowering time was marginally significant (Table 3 ). Specifically, while there was little effect of photoperiod on flowering time in the control treatment, the short photoperiod delayed flowering time by about 2.5 days in the hypomethylation treatment (Figure 2). This suggests that altering the epigenome affected plant response to differing 
TABLE 1: Cross-population germination analyses.

(a) ANOVA

\begin{tabular}{lcccc}
\hline factor & df, residual df & mean squares & f-ratio & p-value \\
\hline Population & 2,172 & 31.698 & 27.948 & $0.001 * * *$ \\
5-AzaC & 1,173 & 0.002 & 0.002 & 0.968 \\
Population $*$ 5-AzaC & 2,172 & 6.69 & 6.226 & $0.002 * *$ \\
\hline
\end{tabular}

(b) Planned orthogonal contrast

\begin{tabular}{lcccc}
\hline comparison & estimate & std. error & t-value & p-value \\
\hline Biennials & -0.06428 & 0.03343 & -1.923 & $0.0557 \dagger$ \\
Fast Plants & 0.07184 & 0.03334 & 2.155 & $0.0322 *$ \\
\hline
\end{tabular}

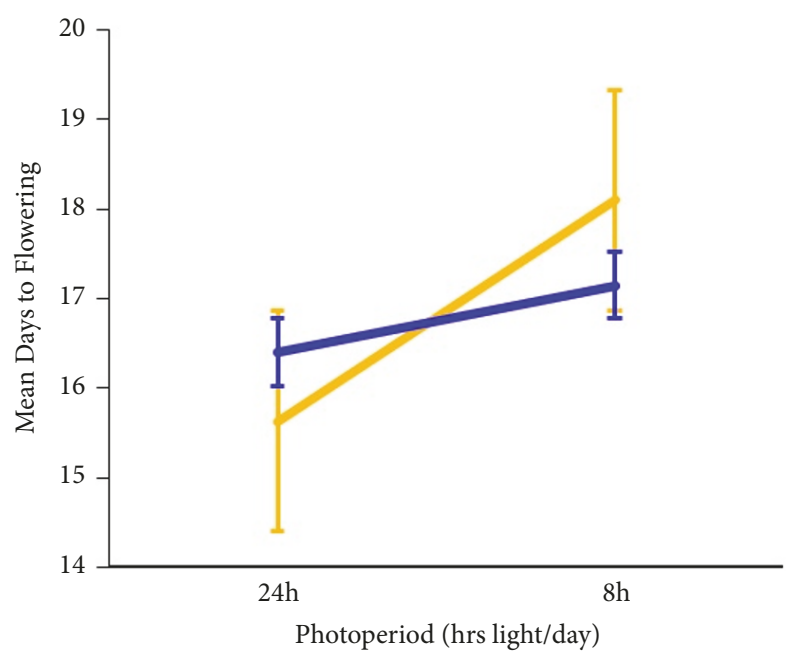

FIGURE 2: Interaction effect between photoperiod and demethylation on flowering time in B. rapa Fast Plants. Yellow indicates individuals under 5-azacytidine hypomethylation treatment (“Aza”), while blue indicates control individuals (“Con”). Error bars represent $1 \mathrm{SE}$ above and below the mean.

light availability, in this case with hypomethylation increasing plasticity. (Note for use of ANOVA: homogeneity of variance was violated for flowering time. However, the difference is just under the threshold for significance, and ANOVA is generally robust to minor violations of the homoscedasticity assumption [38].) Methylation did not affect number of leaves or leaf length but significantly reduced leaf width and number of flowers (Table 2; Figure 3). The hypomethylation treatment reduced leaf width by about $22 \%$ and reduced fitness, based on number of flowers, by approximately $30 \%$ (Figure 3 ).

\section{Discussion}

In this study, we show that treatment of seeds with 5azacytidine affects several plant traits at different life history stages in different populations of Brassica rapa, and that these effects can differ among populations and depending on environmental conditions such as photoperiod. 5-Azacytidine is a well-established demethylation agent [29, 30, 39] with a known mechanism of activity [28] that has previously been used in a number of studies to cause global demethylation in seeds $[12,26,31,32,34-36,39]$. It has been shown to demethylate DNA globally at a higher level than the comparable Zebularine, including transposable element regions that would normally be silenced [39]. Still, studies conducted in mammalian systems have brought to light important evidence that 5-azacytidine and similar demethylating agents can have additional unintended chemical effects. It has been theorized that cytotoxic effects observed in 5-azaC treated mice lines are due to covalent binding of DNA methyltransferase to 5-azaC rather than hypomethylation [33] and that this binding can cause an increased risk of mutations [37]. Additionally, 5-azacytidine can be incorporated into RNA and this may incur additional adverse effects due to transcriptomic changes occurring haphazardly in different parts of the genome [40]. 5-Azacytidine has also been shown to elicit changes to root system development [41] potentially due to one of these alternate mechanisms. However, the different effects we observed under different environmental conditions suggest that general cytotoxicity is unlikely to be the sole cause of our observed results. Because of this, we proceed with the assumption that DNA demethylation was involved in the effects we observed. But we do acknowledge that other side-effects of 5-azacytidine administration cannot be ruled out as potential contributing factors.

Previous studies using 5-azaC have found that demethylation influences a variety of traits, including shade-avoidance [29], premature ripening [42], and somatic embryogenesis [30]. Since it is thought that one of the main effects of methylation is the suppression of transposable elements (TEs), and TEs influence a variety of phenotypes in different ways, it is not surprising that the effects of 5-azaC are widespread and unpredictable [8].

In our experiment, we found that treatment with 5-azaC influenced the timing of germination, and that the effects differed for populations differing in life history characteristics. Recent studies of germination in Arabidopsis have shown that the major DNA methylation changes occur during the germination process $[8,43]$ and that changes in germination rate are impacted by histone demethylation [44]. Germination is a critical life history trait, given that germinating at the wrong time under the wrong conditions can be fatal. Although the effect of demethylation on germination was relatively small, causing differences of only about a day, 


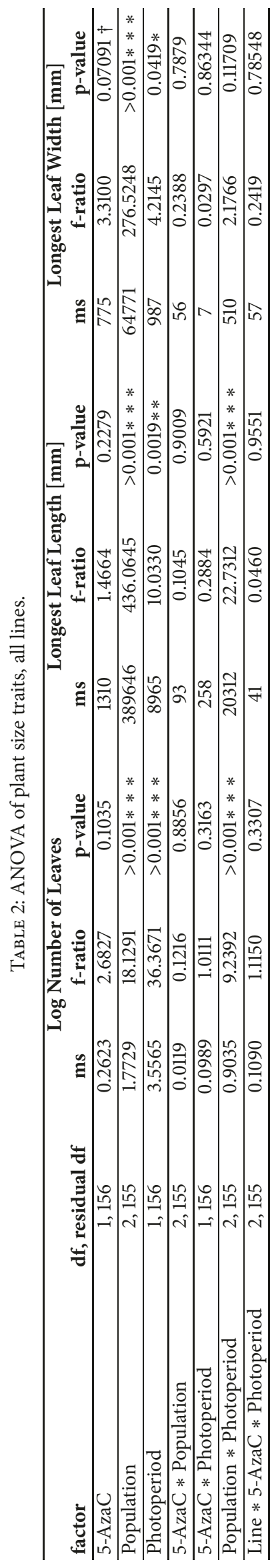




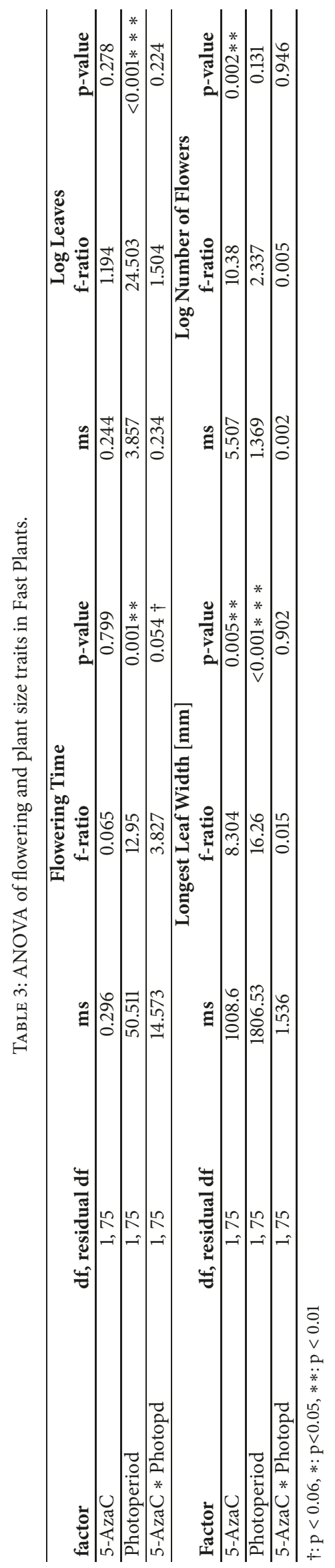



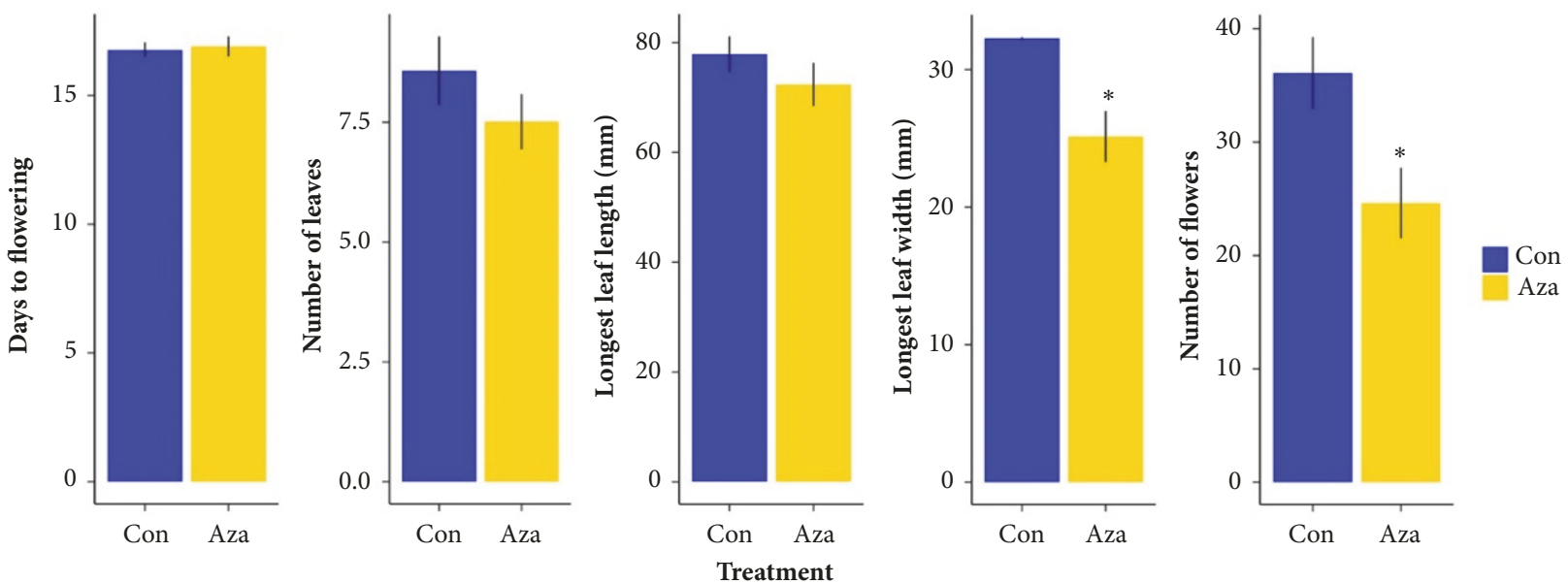

FIGURE 3: Differences in phenotypic traits between 5-AzaC treated and untreated B. rapa Fast Plants. Blue bars indicate control individuals ("Con"), while yellow bars denote individuals under 5-azacytidine hypomethylation treatment ("Aza"). Leaf size measurements are in mm. Error bars represent $1 \mathrm{SE}$ above and below the mean. Significance quantified by planned orthogonal contrast: $* *(.001<\mathrm{p}<.01)$.

this can still be biologically significant, since seedlings with even a one day head start could potentially overtop and outcompete neighbors. In any case, our results support the idea that epigenetic effects can influence germination. The fact that the different populations differed in the effects of hypomethylation on germination suggests that there may be epigenetic differences underlying the different populations.

As in the study by Bossdorf et al. [12] in Arabidopsis thaliana, our studied populations demonstrated variation in the response to the 5-azaC treatment. We found that 5azaC influenced the expression of some adult plant size and flowering traits, but that this effect was only present in the Fast Plant population and varied depending on photoperiod. In the Fast Plant population, we found that 5-azaC reduced leaf width and number of flowers by a substantial amount. This result is consistent with the overall trend of reduced plant growth traits and fitness in 5-AzaC treated individuals in Bossdorf et al. [12]. The administration of demethylating agents has also been shown to reduce plant growth in Taraxacum officinale [45], and DNA methylation has been linked to variation in other leaf traits [46]. Although it is possible that off-target effects of 5-AzaC such as mutagenesis could have played a role, it appears that the results support the idea that methylation patterns are important for plant growth, and that disrupting these patterns results in a loss of fitness, which has important consequences for the evolution of plant traits.

The 5-azaC treatment did not eliminate the vernalization requirement in the biennial population, as was seen in previous studies with the plants Thlaspi arvense [35] and Linum usitatissimum (Fields 1994). It is possible that the lack of an effect was due to the 5 -azaC concentration we used in our experiment. Fieldes' 1994 study administered various concentrations of 5 -azaC to vernalization-dependent populations of L. usitatissimum and found that some concentrations produced the same flowering effect as vernalization, but that concentrations higher than $250 \mu \mathrm{M}$ failed to produce flowering the way that lower concentrations did [34]. A previous study generating hypomethylation in $B$. rapa using 5 -azaC did not use a vernalization-dependent population [26], so whether a different concentration of 5 -azaC would remove the vernalization requirement in biennial $B$. rapa is not known and could be the subject of future investigations.

One intriguing outcome of our experiment was the results for flowering time in the Fast Plant variety. We saw no significant effect of 5-azaC on flowering time, a significant effect of photoperiod on the trait, and a marginally significant interaction between the two (Table 3). The fact that there was no main effect of hypomethylation on flowering time is somewhat surprising given the fact epigenetics plays a key role in the regulation of flowering time [47], and that 5-azaC hypomethylation has previously been shown to alter this trait in Arabidopsis thaliana [12]. While this may appear to present conflicting evidence, a closer look at the populations assessed in the respective studies reveals a more nuanced picture. Of the 22 genotypes used in Bossdorf et al. [12] study, all but two were derived from natural populations, and only one of the two artificial populations made it into the final analysis. Artificial selection may have a role mediating epigenetic impacts. The B. rapa Fast Plant cultivar we used has been bred for short generation time in order to increase their utility as a lab study organism. It is possible that the strong artificial selection for early flowering time in Fast Plants reduced the role of epigenetic regulation of flowering time. Studies of $A$. thaliana found that epigenetic regulation of flowering time is particularly important through the vernalization pathway $[48,49]$, and vernalization is not necessary for flowering in the Fast Plant variety of B. rapa. In addition, we saw that $100 \%$ of Fast Plants that survived transplantation grew to flower during the course of our study, regardless of methylation treatment. This contrasts sharply with the results of Amoah et al. [26], who found a much lower rate of mature individuals flower in the 5 -azaC demethylated population of $B$. rapa which they generated from a $5 \mathrm{mM}$ concentration. This provides further evidence that flowering time in artificially 
selected $B$. rapa populations is less influenced by epigenetic and other environmental factors than this trait is in plant populations that have not been subjected to artificial selection for early flowering.

We found a marginally significant $(\mathrm{p}=0.054)$ interaction effect between methylation and photoperiod treatments for flowering time in Fast Plants. No interaction effects were observed for other traits. As shown in Figure 2, chemically demethylated individuals exhibit a greater difference in mean days to flowering time between photoperiod treatments, with later flowering in treated versus control under short daylength conditions. This result supports the hypothesis that methylation may influence responses to harsher environmental conditions. Several prior studies have explored how epigenetic variation is correlated with and in some cases induces plasticity in response to variation in factors such as nutrient availability and drought $[45,50,51]$. Bossdorf et al. [12] found that 5-azaC increased phenotypic plasticity in traits such as flowering time to environmental variation in nutrients. The groundbreaking work of Herrera et al. [46] demonstrated quantitatively that methylationcontrolled plasticity in resource use became more critical as environmental conditions became harsher. Here we also show that methylation may influence plasticity, in this case plastic response of flowering time to environmental variation in photoperiod. The potential for artificial selection to limit the possible contribution of variable DNA methylation on flowering makes this interaction effect even more intriguing. Even in this highly artificially selected population, the 5-azaC treated plants respond to differential light cues and demonstrate greater variation than control plants. Thus epigenetic modifications may be important in influencing plant traits as well as plasticity, showing that epigenetic patterns can influence responses of plant populations to environmental variation both within and across generations.

\section{Conclusions}

In this study we demonstrated that treatment with 5azacytidine, a demethylating agent, had effects on plant traits at different life history stages in plant populations with differing life-histories. We also showed that this treatment increased phenotypic plasticity in response to light conditions, even in a population that has been bred for rapid flowering. These results contribute to the body of literature investigating how epigenetic modifications such as DNA methylation can affect plasticity and evolution. Continued work in this field can further elucidate the relationship between genetic variation, epigenetic variation, life history traits, and response to more environmental conditions such as temperature, humidity, and soil type. This can be done in both lab-generated demethylated populations, such as those used in this study, and naturally epigenetically variant populations or individuals of the same population grown under differential conditions. Although we are still far from fully understanding the role of epigenetic variation in an organism's response to this environment, this work is crucial to understanding how natural populations will adapt as our world undergoes rapid changes in climate.

\section{Data Availability}

The data used to support the findings of this study are available from the corresponding author upon request.

\section{Conflicts of Interest}

The authors declare that they have no conflicts of interest.

\section{References}

[1] S. J. Franks and A. A. Hoffmann, "Genetics of climate change adaptation," Annual Review of Genetics, vol. 46, pp. 185-208, 2012.

[2] C. L. Richards, O. Bossdorf, and M. Pigliucci, "What role does heritable epigenetic variation play in phenotypic evolution?" Bioscience, vol. 60, no. 3, pp. 232-237, 2010.

[3] Q. Gan, T. Yoshida, O. G. McDonald, and G. K. Owens, "Concise review: epigenetic mechanisms contribute to pluripotency and cell lineage determination of embryonic stem cells," Stem Cells, vol. 25, no. 1, pp. 2-9, 2007.

[4] E. V. A. Jablonka and G. A. L. Raz, "Transgenerational epigenetic inheritance: prevalence, mechanisms, and implications for the study of heredity and evolution," The Quarterly Review of Biology, vol. 84, no. 2, pp. 131-176, 2009.

[5] F. Mohn and D. Schübeler, "Genetics and epigenetics: stability and plasticity during cellular differentiation," Trends in Genetics, vol. 25, no. 3, pp. 129-136, 2009.

[6] P. W. Laird, "Principles and challenges of genome-wide DNA methylation analysis," Nature Reviews Genetics, vol. 11, no. 3, pp. 191-203, 2010.

[7] T. Suganuma and J. L. Workman, "Signals and combinatorial functions of histone modifications," Annual Review of Biochemistry, vol. 80, pp. 473-499, 2011.

[8] T. Kawakatsu, J. R. Nery, R. Castanon, and J. R. Ecker, “Dynamic DNA methylation reconfiguration during seed development and germination," Genome Biology, vol. 18, no. 1, 2017.

[9] O. Bossdorf, C. L. Richards, and M. Pigliucci, "Epigenetics for ecologists," Ecology Letters, vol. 11, no. 2, pp. 106-115, 2008.

[10] H. J. Kilvitis, M. Alvarez, C. M. Foust, A. W. Schrey, M. Robertson, and C. L. Richards, "Ecological epigenetics," Advances in Experimental Medicine and Biology, vol. 781, pp. 191-210, 2014.

[11] I. Kronholm and S. Collins, "Epigenetic mutations can both help and hinder adaptive evolution," Molecular Ecology, vol. 25, no. 8, pp. 1856-1868, 2016.

[12] O. Bossdorf, D. Arcuri, C. L. Richards, and M. Pigliucci, "Experimental alteration of DNA methylation affects the phenotypic plasticity of ecologically relevant traits in Arabidopsis thaliana," Evolutionary Ecology, vol. 24, no. 3, pp. 541-553, 2010.

[13] C. E. Niederhuth, "Widespread natural variation of DNA methylation within angiosperms," Genome Biology, vol. 17, p. 194, 2016.

[14] Z. Lippman, A.-V. Gendrel, M. Black et al., "Role of transposable elements in heterochromatin and epigenetic control," Nature, vol. 430, no. 6998, pp. 471-476, 2004.

[15] M. M. Suzuki and A. Bird, "DNA methylation landscapes: provocative insights from epigenomics," Nature Reviews Genetics, vol. 9, no. 6, pp. 465-476, 2008.

[16] A. Pecinka, A. Abdelsamad, and G. T. H. Vu, "Hidden genetic nature of epigenetic natural variation in plants," Trends in Plant Science, vol. 18, no. 11, pp. 624-632, 2013. 
[17] Z. J. Chen, M. Ha, and D. Soltis, "Polyploidy: Genome obesity and its consequences," New Phytologist, vol. 174, no. 4, pp. 717720, 2007.

[18] M. J. Dubin, P. Zhang, D. Meng et al., "DNA methylation in Arabidopsis has a genetic basis and shows evidence of local adaptation," eLife, vol. 4, 2015.

[19] J. Peñuelas and I. Filella, "Phenology: Responses to a warming world," Science, vol. 294, no. 5543, pp. 793-795, 2001.

[20] C. Parmesan and G. Yohe, "A globally coherent fingerprint of climate change impacts across natural systems," Nature, vol. 421, no. 6918, pp. 37-42, 2003.

[21] S. J. Franks, J. J. Weber, and S. N. Aitken, "Evolutionary and plastic responses to climate change in terrestrial plant populations," Evolutionary Applications, vol. 7, no. 1, pp. 123-139, 2014.

[22] S. J. Franks, S. Sim, and A. E. Weis, "Rapid evolution of flowering time by an annual plant in response to a climate fluctuation," Proceedings of the National Acadamy of Sciences of the United States of America, vol. 104, no. 4, pp. 1278-1282, 2007.

[23] S. J. Franks, N. C. Kane, N. B. O’Hara, S. Tittes, and J. S. Rest, "Rapid genome-wide evolution in Brassica rapa populations following drought revealed by sequencing of ancestral and descendant gene pools," Molecular Ecology, vol. 25, no. 15, pp. 3622-3631, 2016.

[24] M. R. G. Khan, X.-Y. Ai, and J.-Z. Zhang, "Genetic regulation of flowering time in annual and perennial plants," Wiley Interdisciplinary Reviews: RNA, vol. 5, no. 3, pp. 347-359, 2014.

[25] P. H. Williams and C. B. Hill, "Rapid-cycling populations of Brassica," Science, vol. 232, no. 4756, pp. 1385-1389, 1986.

[26] S. Amoah, S. Kurup, C. M. Rodriguez Lopez et al., "A Hypomethylated population of Brassica rapa for forward and reverse Epi-genetics," BMC Plant Biology, vol. 12, article no. 193, 2012.

[27] L. Ji, D. A. Neumann, and R. J. Schmitz, "Crop epigenomics: Identifying, unlocking, and harnessing cryptic variation in crop genomes," Molecular Plant, vol. 8, no. 6, pp. 860-870, 2015.

[28] P. A. Jones, "Effects of 5-azacytidine and its 2I-deoxyderivative on cell differentiation and DNA methylation," Pharmacology \& Therapeutics, vol. 28, no. 1, pp. 17-27, 1985.

[29] G. S. Tatra, J. Miranda, C. C. Chinnappa, and D. M. Reid, "Effect of light quality and 5-azacytidine on genomic methylation and stem elongation in two ecotypes of Stellaria longipes," Physiologia Plantarum, vol. 109, no. 3, pp. 313-321, 2000.

[30] H. P. F. Fraga, L. N. Vieira, C. A. Caprestano et al., "5-Azacytidine combined with 2,4-D improves somatic embryogenesis of Acca sellowiana (O. Berg) Burret by means of changes in global DNA methylation levels," Plant Cell Reports, vol. 31, no. 12, pp. 2165-2176, 2012.

[31] H. Kondo, H. Ozaki, K. Itoh, A. Kato, and K. Takeno, "Flowering induced by 5-azacytidine, a DNA demethylating reagent in a short-day plant, Perilla frutescens var. crispa," Physiologia Plantarum, vol. 127, no. 1, pp. 130-137, 2006.

[32] H. Sano, I. Kamada, S. Youssefian, M. Katsumi, and H. Wabiko, "A single treatment of rice seedlings with 5-azacytidine induces heritable dwarfism and undermethylation of genomic DNA," MGG Molecular \& General Genetics, vol. 220, no. 3, pp. 441-447, 1990.

[33] R. Jüttermann, E. Li, and R. Jaenisch, "Toxicity of 5-aza-2ıdeoxycytidine to mammalian cells is mediated primarily by covalent trapping of DNA methyltransferase rather than DNA demethylation," Proceedings of the National Acadamy of Sciences of the United States of America, vol. 91, no. 25, pp. 11797-11801, 1994.

[34] M. A. Fieldes, "Heritable effects of 5-azacytidine treatments on the growth and development of flax (Linum usitatissimum) genotrophs and genotypes," Genome, vol. 37, no. 1, pp. 1-11, 1994.

[35] J. E. Burn, D. J. Bagnall, J. D. Metzger, E. S. Dennis, and W. J. Peacock, "DNA methylation, vernalization, and the initiation of flowering," Proceedings of the National Acadamy of Sciences of the United States of America, vol. 90, no. 1, pp. 287-291, 1993.

[36] R. K. Genger, W. J. Peacock, E. S. Dennis, and E. J. Finnegan, "Opposing effects of reduced DNA methylation on flowering time in Arabidopsis thaliana," Planta, vol. 216, no. 3, pp. 461466, 2003.

[37] J. K. Christman, "5-Azacytidine and 5-aza-2'-deoxycytidine as inhibitors of DNA methylation: mechanistic studies and their implications for cancer therapy," Oncogene, vol. 21, no. 35, pp. 5483-5495, 2002.

[38] G. V. Glass, P. D. Peckham, and J. R. Sanders, "Consequences of failure to meet assumptions underlying the fixed effects analyses of variance and covariance," Review of Educational Research, vol. 42, no. 3, pp. 237-288, 1972.

[39] P. T. Griffin, C. E. Niederhuth, and R. J. Schmitz, "A comparative analysis of 5-azacytidine-and zebularine-induced DNA demethylation," G3: Genes, Genomes, Genetics, vol. 6, no. 9, pp. 2773-2780, 2016.

[40] P. W. Hollenbach, A. N. Nguyen, H. Brady et al., "A comparison of azacitidine and decitabine activities in acute myeloid leukemia cell lines," PLoS ONE, vol. 5, no. 2, p. e9001, 2010.

[41] T. Kanchanaketu and V. Hongtrakul, "Treatment of 5azacytidine as DNA demethylating agent in Jatropha curcas L," Kasetsart Journal - Natural Science, vol. 49, no. 4, pp. 524-535, 2015.

[42] S. Zhong, Z. Fei, Y.-R. Chen et al., "Single-base resolution methylomes of tomato fruit development reveal epigenome modifications associated with ripening," Nature Biotechnology, vol. 31, no. 2, pp. 154-159, 2013.

[43] R. Narsai, Q. Gouil, D. Secco et al., "Extensive transcriptomic and epigenomic remodelling occurs during Arabidopsis thaliana germination," Genome Biology, vol. 18, no. 1, 2017.

[44] J.-N. Cho, J.-Y. Ryu, Y.-M. Jeong et al., "Control of seed germination by light-induced histone arginine demethylation activity," Developmental Cell, vol. 22, no. 4, pp. 736-748, 2012.

[45] K. J. F. Verhoeven and T. P. van Gurp, "Transgenerational effects of stress exposure on offspring phenotypes in apomictic dandelion," PLoS ONE, vol. 7, no. 6, 2012.

[46] C. M. Herrera, M. I. Pozo, and P. Bazaga, "Jack of all nectars, master of most: DNA methylation and the epigenetic basis of niche width in a flower-living yeast," Molecular Ecology, vol. 21, no. 11, pp. 2602-2616, 2012.

[47] F. Bratzel and F. Turck, "Molecular memories in the regulation of seasonal flowering: From competence to cessation," Genome Biology, vol. 16, no. 1, article no. 192, 2015.

[48] R. Amasino, "Vernalization, competence, and the epigenetic memory of winter," The Plant Cell, vol. 16, no. 10, pp. 2553-2559, 2004.

[49] D. Kim and S. Sung, "Genetic and epigenetic mechanisms underlying vernalization," The Arabidopsis Book, vol. 12, p. e0171, 2014.

[50] A. Boyko and I. Kovalchuk, "Genome instability and epigenetic modification-heritable responses to environmental stress?" 
Current Opinion in Plant Biology, vol. 14, no. 3, pp. 260-266, 2011.

[51] Y.-Y. Zhang, M. Fischer, V. Colot, and O. Bossdorf, "Epigenetic variation creates potential for evolution of plant phenotypic plasticity," New Phytologist, vol. 197, no. 1, pp. 314-322, 2013. 


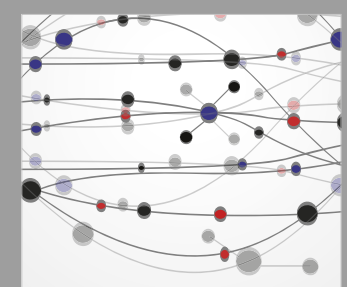

The Scientific World Journal
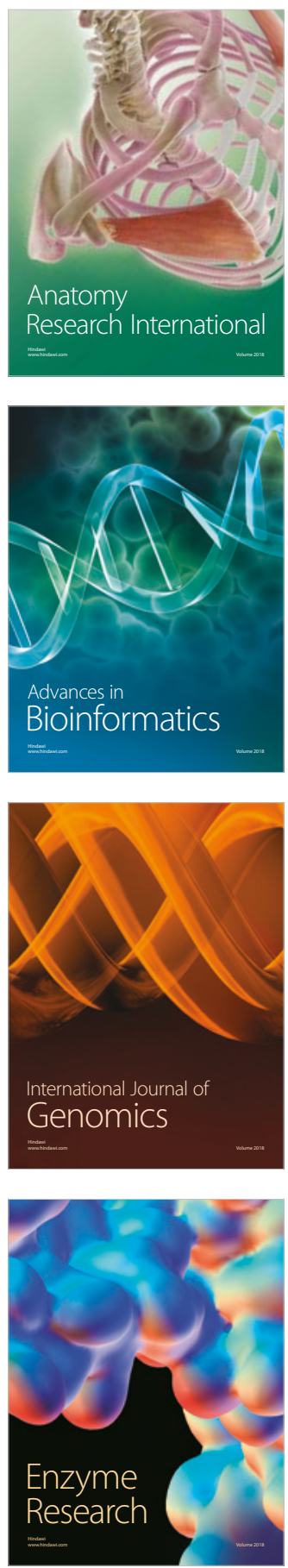
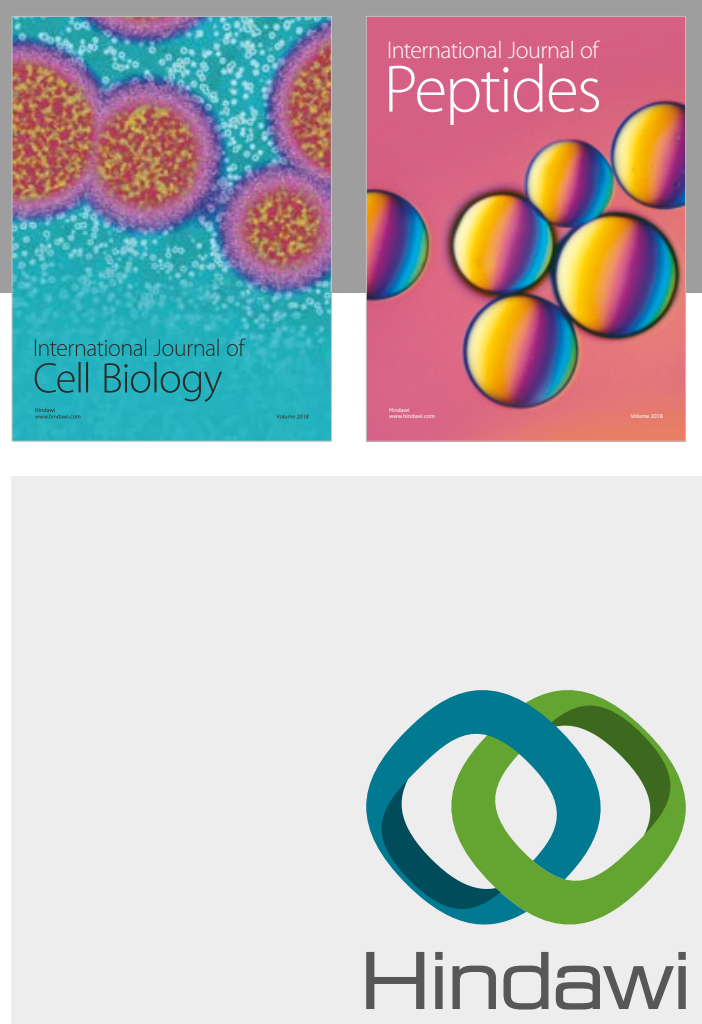

Submit your manuscripts at

www.hindawi.com
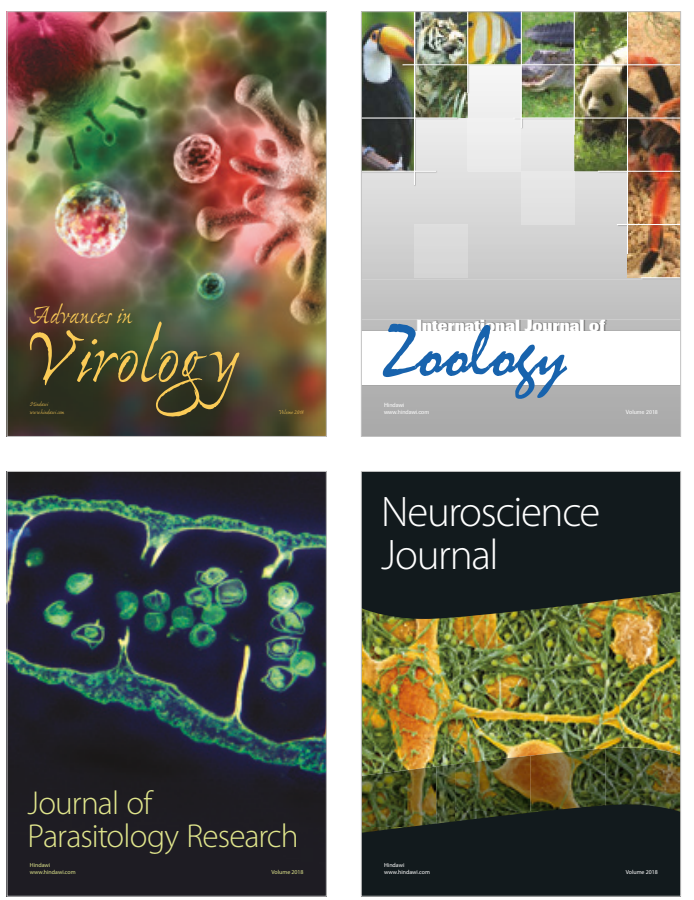
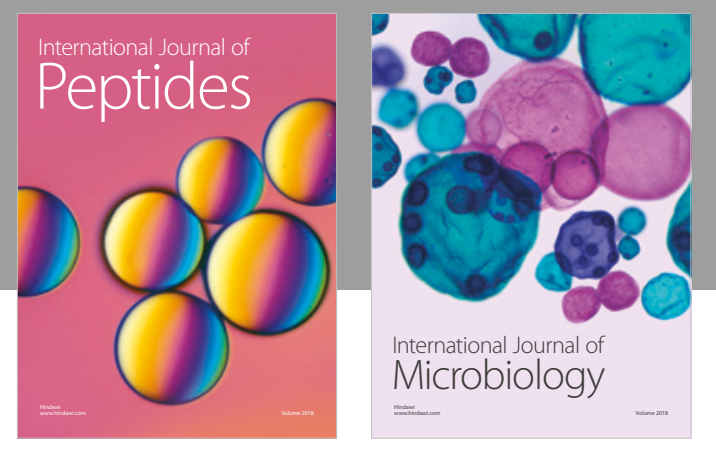

nternational Journal of Microbiology
Journal of
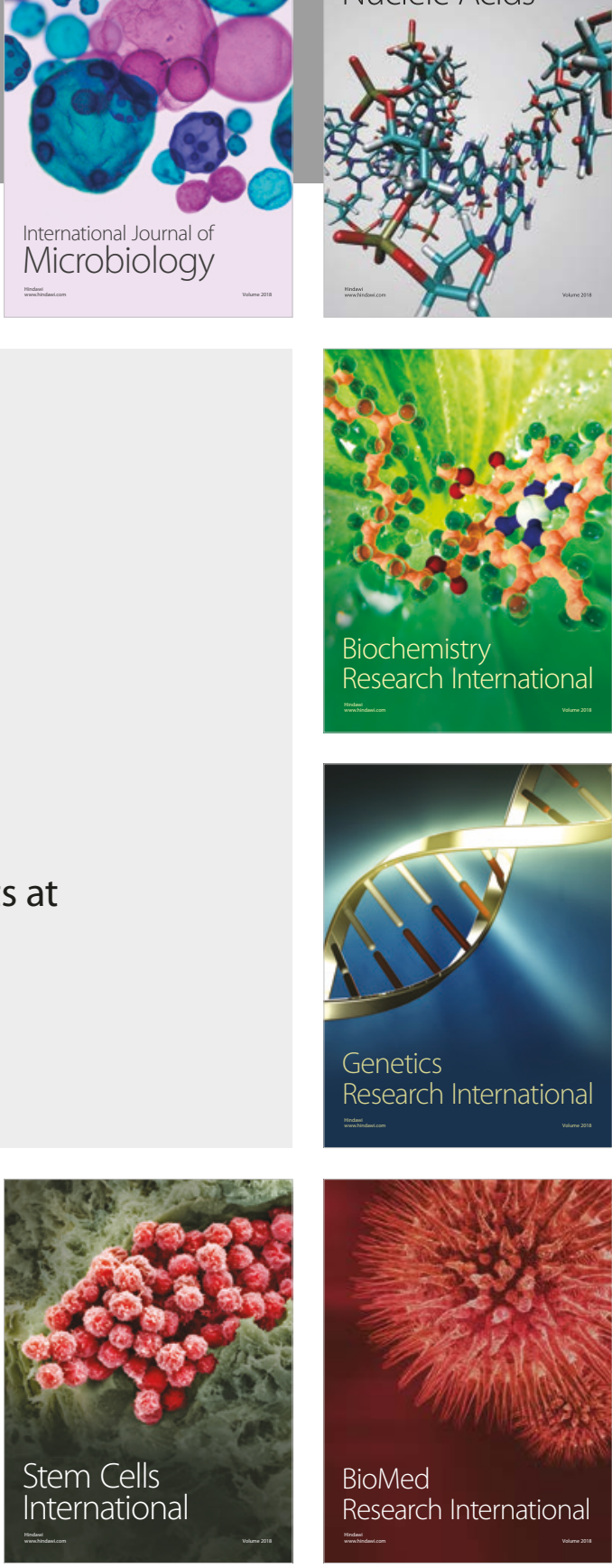
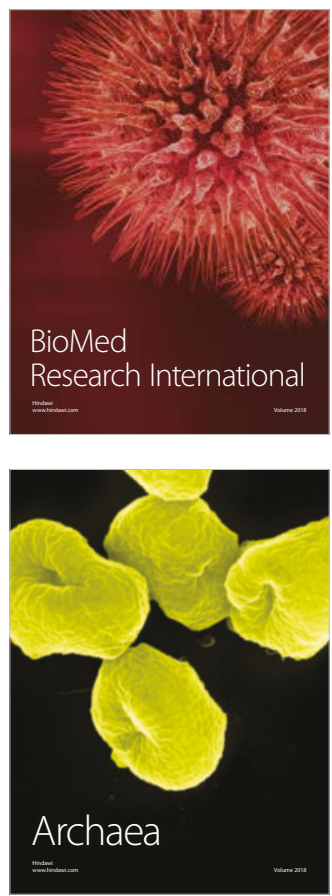